\title{
Formative Evaluation in Document Design: Validity of the Plus-Minus Method For the Pretesting of Public Information Brochures
}

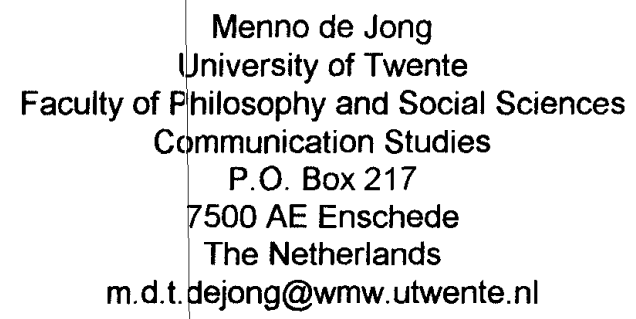

This article provides empirical data on the validity of the plus-minus method for the pretesting of public information brochures. Six brochures were tested using the plus-minus method, and revised on the basis of the pretest results. A series of experiments comparing original and revised versions of the brochures showed that the reader problems detected were valuable ones: Both the target readers' appreciation of the brochures and the effectiveness of the brochures themselves showed a significant improvement. Another study in which experts judged the importance of the problems detected confirmed these findings, but also raised doubts about the extent to which individual revisers can reliably judge the importance of reader problems.

\section{Introduction}

In the literature on professional communication, there has been a noticeable increase in the attention paid to reader-focused evaluation techniques for use during the design process of documents. The assumption that professional writers are capable of judging a document's quality from the reader's point of view has been frequently disproved - informally in practice, but also in ernpirical research (e.g., De Jong and Lentz 1996; Lentz and De Jong 1997). However, little is known yet about the merits and restrictions of the various methods available for reader-focused text evaluation (see De Jong and Schellens 1997 for an overview).

This article describes a series of studies ${ }^{1}$ on the validity of a reader-focused method for use in the evaluation of various types of documents: the plus-minus method. This is a pretest approach frequently used in public information practice in the Netherlands. The method involves asking participants to read a text from start to finish and to mark their positive and negative reading experiences with pluses and minuses, respectively, in the margin. Pluses and minuses may be assigned to all sorts of text elements (from chapters to words) and for various reasons (e.g., comprehensibility, appreciation, relevance of the information). After that, individual interviews are held, focusing on the reasons for every plus and minus ${ }^{2}$. The interviews result in a list of 


\section{A Contemporary Renaissance: Changing the Way We Communicate}

reader problems, actual or potential, which then forms the input for a subsequent round of revisions. Normally, the plus-minus method is followed by a short semi-structured questionnaire focusing on the macrostructure and a general evaluation of the text.

The plus-minus method is an approach that relies on participants' self-reports, rather than on any assessment or observation of their performance. This may have disadvantageous effects on the validity of the results. Readers may be unaware of the comprehension problems they have, or, conversely, may mention problems they do not really have in an attempt to please the facilitator. On the other hand, the method is capable of detecting a wide variety of reader problems - a much broader range of problems than current approaches of usability testing - and of providing detailed diagnostic information without unduly disturbing the reading process of participants.

The validity of the plus-minus method was investigated using six public information brochures. Three of these brochures were predominantly informative or instructional (dealing with rent subsidies, counseling services available to crime victims, and income tax), while three others were primarily persuasive (two dealing with alcohol abuse, and a third dealing with safe sex). The validity of the method was addressed in two complementary ways. First, the importance of the reader feedback was investigated with revision as an intermediate step. The key question here was whether a revision on the basis of the reader feedback would lead to an improvement in the effectiveness of the brochures. Second, with respect to four of the brochures, expert judgments were collected on the importance of the reader problems.

\section{Six revision-based validation experiments}

In all six brochures, the pretests invariably brought to light a large number of potential reader problems (varying between 107 and 188 different problems per brochure). The problems related not only to the comprehensibility and applicability of the information given in the brochures, but also, for instance, to the acceptability of the information, or readers' appreciation of presentation characteristics. In the subsequent revision phase, 43 to 72 percent of the reader problems led to textual changes. It may therefore be said that the pretests resulted in considerable changes in the six brochures. The question to be answered in the follow-up experiments was whether these textual changes had a favorable effect on the quality of the brochures. This question was investigated in two complementary ways:

- A series of motivated-choice experiments focused on the preference of target readers with regard to relatively small brochure units (e.g., paragraphs). Participants (between 30 and 40 per brochure) were presented with original and revised brochure fragments side by side, and were asked to express a preference for one of the versions of each fragment, using a five-point scale.

- A series of independent-groups experiments focused on the effectiveness of the brochure versions and on the target readers' overall assessment of the brochures. Half the participants received the original brochure, and the other half the revised version. Between 60 and 80 target readers participated in the investigation of each brochure. The main dependent variables were comprehensibility (in the case of all brochures), persuasiveness (in the case of the three brochures with persuasive aims), and overall assessment (in the case of all brochures).

\section{0-7803-4890-7/98/\$10.00 ๑ 1998 IEEE}




\section{A Contemporary Renaissance: Changing the Way We Communicate}

\section{Motivated-choice experiments}

Table 1 shows the results of the motivated-choice experiments. The table represents the mean scores of the participants for all brochure units, with " 1 ". representing a preference for the original brochure and " 5 " a preference for the revision. The statistical significance of the results was assessed using 95\% confidence intervals: If an interval is entirely below the neutral score of " 3 ", the original version is significantly better than the revised one $(p .05)$; if the interval is entirely above the neutral score, the readers find the revised version is significantly better.

\begin{tabular}{l|cc} 
& & \\
\hline Brochure & Mean score & $95 \%$ Confidence interval \\
\hline Rent Subsidy & 3.71 & $3.59-3.83$ \\
Victim Aid & 3.60 & $3.52-3.68$ \\
Hirst Job & 3.51 & $3.39-3.63$ \\
Alcohol 1 & 3.17 & $3.09-3.25$ \\
Alcohol 2 & 3.26 & $3.17-3.37$ \\
Safe Sex & 3.31 & $3.25-3.37$
\end{tabular}

Table 1. Mean Scores of the Motivated-Choice Experiments

(Note: Scores on a five-point scale; $1=$ preference for the original version, $5=$ preference for the revision)

In all the motivated-choice experiments, readers showed a significant preference for the revised versions of the brochures. That means that the pretests and subsequent revisions certainly had a positive effect on the target audience's appreciation of the brochures in question. However, their preference was clearer for the three informative and instructional brochures than for the persuasive ones. Apparently, it is easier to achieve univocal improvements in the case of problems concerning the usability of information than for problems concerning readers' affective reactions.

\section{Independent-groups experiments}

Table 2 and 3 present the results of the experiments regarding the comprehensibility of the information in the brochures. In all cases, participants were given a set of comprehension and application questions about the information in the brochures. In the case of the three informative and instructional brochures, they had to answer the questions by using the text. The statistical significance of the results was tested using one-tailed t-tests. In the case of the three persuasive brochures, they had to answer the questions without reference to the text, before and after reading the brochure. The statistical significance was tested using a repeated measures analysis of variance.

\begin{tabular}{l|ccl}
\hline Brochure & $\begin{array}{c}\text { Original } \\
\text { Version }\end{array}$ & $\begin{array}{c}\text { Revised } \\
\text { version }\end{array}$ & Significance of the results \\
\hline Rent Subsidy (24 questions) & 15.72 & 18.00 & $\mathrm{p}<.005 ;$ Cohen's d $=.70$ \\
Victim Aid (40 questions) & 23.57 & 23.93 & n.s. \\
First Job (15 questions) & 11.00 & 11.87 & $\mathrm{p}<.05 ;$ Cohen's d $=.44$ \\
\hline
\end{tabular}

Table 2. Comprehensibility of the three informative/instructional brochures

(Note: Mean number of correct answers) 


\section{A Contemporary Renaissance: Changing the Way We Communicate}

\begin{tabular}{lccl}
\hline Brochure & $\begin{array}{l}\text { Original } \\
\text { version }\end{array}$ & $\begin{array}{c}\text { Revised } \\
\text { version }\end{array}$ & Significance of the results \\
\hline Alcohol l & 1.57 & 1.70 & n.s. \\
Alcohol 2 & 3.67 & 3.55 & n.s. \\
Safe Sex & 2.02 & 3.28 & $\mathbf{p}<.05 ; \mathrm{eta}^{2}=.07$ \\
\hline
\end{tabular}

Table 3. Comprehensibility of the three persuasive brochures

(Note: Differences between the number of correct answers before and after reading the brochures)

In the case of three brochures, a significant improvement was found in the comprehensibility of the information. The effect sizes of the observed differences - as indicated by Cohen's $d$ and eta $^{2}$, respectively (cf. Cohen 1977) - varied from rather large (Rent Subsidy) to medium (First Job and Safe Sex). Taken together (by means of Z-scores), the results of the six experiments indicate a significant improvement in the comprehensibility of the brochures (one-tailed t-test, $t=-2.867, d f=406.122, p<.005)$. However, the overall effect size of the difference was rather small (Cohen's $d$.28).

Table 4 presents the results regarding the persuasiveness of the three persuasive brochures. This was measured using attitude questionnaires before and after exposure to the brochures. These questionnaires required participants to react to assertions about alcohol or safe sex on nine-point scales, from "Strongly agree" to "Strongly disagree". The significance of the results was tested using a repeated measures analysis of variance.

\begin{tabular}{lccl} 
Brochure & $\begin{array}{c}\text { Original } \\
\text { version }\end{array}$ & $\begin{array}{c}\text { Revised } \\
\text { version }\end{array}$ & Significance of the results \\
\hline Alcohol l & .04 & .34 & $\mathrm{p}<.05 ; \mathrm{eta}^{2}=.05$ \\
Alcohol 2 & .03 & .23 & n.s. \\
Safe Sex & .04 & .37 & $\mathrm{p}<.05 ; \mathrm{eta}^{2}=.07$ \\
\hline
\end{tabular}

Table 4. Persuasiveness of the brochures

(Note: Differences between attitude questions before and after reading the brochures)

In the case of two of the three brochures, the pretest and subsequent revision had a positive effect on the persuasiveness of the information. In both cases, a medium effect was found (as indicated by eta $^{2}$ ). Taken together (with Z-scores), the results of the experiments show a significant overall improvement in the persuasiveness of the brochures $(F(I, 229)=9.534$, $p$.05), and an eta ${ }^{2}$ of .04 , corresponding to a small-to-medium effect.

Finally, Table 5 presents the overall assessment of the original and revised versions of the brochures. In each experiment, participants had to react to assertions about the brochure on scales ranging from "Strongly agree" to "Strongly disagree". The scale size varied between the 


\section{A Contemporary Renaissance: Changing the Way We Communicate}

brochures; for convenience of comparison, the scores of all brochures are recalculated here to correspond to a five-point scale. The statistical significance was tested using a t-test.

\begin{tabular}{lccl}
\hline Brochure & Original version & Revised version & Significance of the results \\
& & & \\
\hline Rent Subsidy & 2.14 & 2.08 & n.s. \\
Victim Aid & 2.03 & 1.70 & $\mathrm{p}<.05$; Cohen's d =.46 \\
First Job & 1.97 & 1.92 & n.s. \\
Alcohol I & 2.28 & 2.30 & n.s. \\
Alcohol 2 & 2.18 & 2.20 & n.s. \\
Safe Sex & 1.34 & 1.41 & n.s. \\
\hline
\end{tabular}

Table 5. Overall assessment of the brochures

(Note: Mean scores on the overall text-evaluation questionnaires ( $1=$ =positive; $5=$ negative)

Although in all motivated-choice experiments readers expressed a significant preference for the revised versions of the brochures, only one independent-groups experiment - relating to the Victim Aid brochure - showed a significant improvement in readers' overall assessment of the brochure, with Cohen's $d$ indicating a medium effect. The improvement in the Victim Aid brochure can be attributed to the drastic changes that were made in its macro-structure: The results particularly suggested an improvement in the overall organization, the ease with which information could be looked up, and the perceived comprehensibility of the information. The general tendency over all brochures (computed with Z-scores), however, did not correspond to a significant difference between the original and revised brochure versions $(t-t e s t, t=624, d f=412$, $p=.533)$.

All in all, the pretests and subsequent revisions proved to be a valuable step in the case of all the brochures involved. The motivated-choice experiments yielded favorable results in relation to all brochures, indicating a predominant preference of the target readers for the revisions. Moreover, in five of the six independent-groups experiments, the pretest and subsequent revision resulted in an improvement in effectiveness with respect to comprehensibility of the information (Rent Subsidy, First Job and Safe Sex), persuasiveness (Alcohol 1 and Safe Sex), or perceived userfriendliness (Victim Aid).

\section{Four expert-judgment validation studies}

For four of the brochures involved in the revision-based experiments - i.e., Rent Subsidy, Victim Aid, Alcohol 2, and Safe Sex - additional data were collected on the importance of the reader problems detected. In these studies, ten experts (five subject-matter experts and five text experts) were asked to judge, using a five-point scale, the importance of all the reader problems detected in a brochure. They were given a general description of each reader problem but were not told how many pretest participants had mentioned it. The resulting additional validation data make it possible to assess the importance of individual problems. Table 7 shows the mean importance ratings given by individual experts.

\section{0-7803-4890-7/98/\$10.00 @ 1998 IEEE}


A Contemporary Renaissance: Changing the Way We Communicate

\begin{tabular}{lccccc}
\hline Brochure & $\begin{array}{c}\text { Score 1 } \\
\text { Important }\end{array}$ & Score 2 & $\begin{array}{c}\text { Score 3 } \\
\text { Neutral }\end{array}$ & Score 4 & $\begin{array}{c}\text { Score 5 } \\
\text { Unimportant }\end{array}$ \\
\hline Rent Subsidy & .19 & .26 & .16 & .21 & .19 \\
Victim Aid & .22 & .20 & .16 & .20 & .22 \\
Alcohol 2 & .16 & .17 & .17 & .22 & .26 \\
Safe Sex & .11 & .18 & .13 & .20 & .37 \\
Mean & .17 & .20 & .15 & .21 & .26 \\
\hline Men & & & & \\
\hline
\end{tabular}

Table 7. Mean proportion of reader problems with different importance ratings by individual experts

As can be seen in Table 7, the participating experts generally confirmed that the pretests had provided valuable feedback on the brochures. On average, individual experts judged 37 percent of the reader problems to be important (scoring 1 or 2). However, they also indicated that not all the problems mentioned by readers were important ones: Almost half the reader problems were in their view unimportant (scoring 4 or 5). Consequently, the sifting of important reader problems must be considered an essential step when using plus-minus data to optimize brochures.

Table 8 presents the combined importance ratings given by the ten experts as a group. Combining the individual expert ratings to one overall score for each reader problem makes it possible to distinguish significantly important and significantly unimportant reader problems (using 95\% confidence intervals).

\begin{tabular}{lccc}
\hline Brochure & $\begin{array}{c}\text { Significantly } \\
\text { important }\end{array}$ & Neutral & $\begin{array}{c}\text { Significantly } \\
\text { unimportant }\end{array}$ \\
\hline Rent Subsidy & .10 & .79 & .10 \\
Victim Aid & .18 & .65 & .16 \\
Alcohol 2 & .06 & .74 & .20 \\
Safe Sex & .04 & .62 & .33 \\
\hline Mean & .10 & .70 & .21 \\
\hline
\end{tabular}

Table 8. Proportion of reader problems with positive, neutral, and negative importance ratings by the experts as a group

The results in Table 8 confirm that all pretests brought to light some important reader problems, but the amount of significantly important reader feedback is rather disappointing. Instead, the overall ratings by the experts are largely indeterminate. The majority of the reader problems (on average, 70 percent) could not be assigned to either the "Significantly important" or the "Significantly unimportant" category. This may be attributed to a lack of agreement among the experts: On average, the ratings of two independent experts had a correlation coefficient of only .23 ; the total range of correlation coefficients between two experts varied from -.15 to .47 .

All in all, the individual expert-judgment data confirmed the usefulness of the pretest results, while the combined ratings at least did not contradict these results. Apparently, the experts agreed with each other that the pretests had yielded a considerable amount of valuable feedback 


\section{A Contemporary Renaissance: Changing the Way We Communicate}

on the brochures, but they did not agree on precisely which reader problems were the valuable ones. This, of course, highlights the problem of revising on the basis of pretest results: How can we reliably clistinguish the important reader problems from the unimportant ones? The lack of agreement in the expert-judgment data suggests that one cannot rely on the judgments of individual revisers: Different experts will vary in the priority they assign to reader problems. Furthermore, another current approach for distinguishing the most important reader problems that of prioritizing them according to the frequency with which they arise in the pretest - also turned out to be questionable: There was no relation between the frequency with which reader problems arose in the pretests (i.e., the number of pretest participants who mentioned them) and the importance of the problems (mean correlation coefficient $=.08$ ).

\section{Conclusions}

The studies reported above indicate that the plus-minus method can be a useful instrument for collecting reader feedback on public information brochures. In more general terms, they show that self-reporting reader comments may be used to some benefit in document design processes. From the revision-based experiments, the conclusion can be drawn that a revision on the basis of the pretest results generally had a positive effect on the audience's appreciation of the brochures (cf. the motivated-choice experiments), and often also led to an improvement in the brochures' effectiveness (cf. the independent-groups experiments). From the expert-judgment studies, the conclusion can be drawn that all experts would have proposed considerable textual changes in reaction to the plus-minus results, although possibly with respect to different aspects of the brochures.

Nonetheless, more research is needed, particularly concerning the revision phase following a pretest. We have carried out a small-scale descriptive study into the revision strategies of five professional writers using "plus-minus" reader feedback (Schellens and De Jong 1997). The results suggest that on the basis of the same pretest results different revisers may do entirely different things. Future research should therefore focus on developing and validating strategies for evaluating the severity of reader problems, and on distinguishing effective from less effective revision strategies for various types of reader problems.

Notes:

1. A more elaborate report of the studies described in this article may be found in De Jong (1998)

2. The pluses and minuses may also be discussed in focus groups; see De Jong \& Schellens (1998) for a comparison of the results of individual and group pretesting.

\section{References}

Cohen, J. Statistical power analysis for the behavioral sciences. Revised edition. New York: Academic Press. 1977.

Jong, M. de. Reader feedback in text design. Validity of the plus-minus method for the pretesting of public information brochures. $\mathrm{PhD}$ dissertation University of Twente. Utrecht Series in Language and Communication 13. Atlanta, GA: Rodopi. 1998.

\footnotetext{
$0-7803-4890-7 / 98 / \$ 10.00 @ 1998$ IEEE
} 


\section{A Contemporary Renaissance: Changing the Way We Communicate}

Jong, M. de, and L.R.Lentz. "Expert judgments versus reader feedback. A comparison of text evaluation techniques. Journal of Technical Writing and Communication." 26(4): 507-519, 1996.

Jong, M. de, and P.J. Schellens. "Reader-focused text evaluation: An overview of goals and methods." Journal of Business and Technical Communication. 11(4): 402-432, 1997.

Jong, M. de, and P.J.,Schellens. "Focus groups or individual interviews? A comparison of text evaluation approaches." Technical Communication. 45(1): 77-88, 1998.

Lentz, L., and M.de Jong. "The evaluation of text quality: Expert-focused and reader-focused methods compared." IEEE Transactions on Professional Communication. 40(3): 224-234, 1997.

Schellens, P.J., and M. de Jong. "Revision of public information brochures on the basis of reader feedback." Journal of Business and Technical Communication. 11(4): 483-501, 1997.

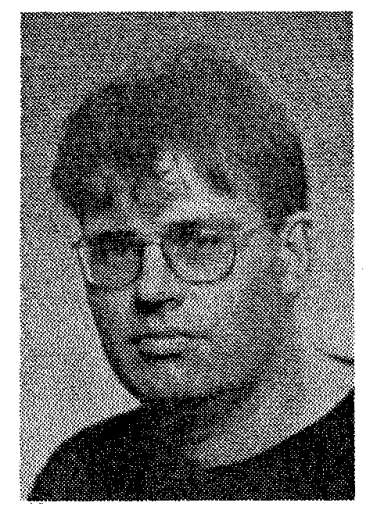

Menno de Jong is an assistant professor of applied linguistics at the University of Twente (Enschede, the Netherlands). His research interests and publications concern the field of formative text evaluation and usability. His Ph.D. dissertation focuses on the value of the plusminus method for the pretesting of public information brochure. His current research focuses on the development and validation of evaluation techniques for World Wide Web sites.

Peter Jan Schellens is a professor of applied linguistics at the University of Twente (Enschede, the Netherlands). He coordinates a research program on verbal communication and text design. His research interests include writing, text evaluation methods and usability. 